\title{
Evaluación de la inteligencia a través de formas cortas del WAIS para poblaciones de nivel socioeconómico bajo*
}

\section{The use of short forms of WAIS for the assessment of the intelligence of persons of low socioeconomic status}

isidora de Andraca**, Cecilia Cobo**, Francisca Rivera**, Fernando Pizarro**

\begin{abstract}
ANDRACA. I. de et al. Evaluación de la inteligencia a través de formas cortas del WAIS para poblaciones de nivel socioeconómico bajo. Rev. Saúde Pública, 27:334-9, 1993. Se propone una versión abreviada de la forma WAIS para evaluar la inteligencia en grupos de población. Se analizaron 161 protocolos completos del WAIS aplicados a mujeres de nivel socioeconómico bajo y se estudió las correlaciones de las distintas subpruebas con el CI total y con los CI verbal y manual. Se seleccionaron seis subpruebas (Comprensióni, Semejanzas, Vocabulario, Cubos, Completación y Ensamblaje) en base a dos criterios: las correlaciones observadas, y las funciones evaluadas en cada subprueba. El análisis de una forma corta con 6 subpruebas y cuatro formas cortas con 4 subpruebas demuestra que todas ellas son adecuadas para la evaluación de la inteligencia como variable de confusión. El error estándar para la estimación del CI total varía entre 3 y 4 puntos, y el error de clasificación corresponde a un $3-7 \%$. Cuando se evalúa inteligencia materna como variable de confusión del desarollo infantil la forma corta que combina Comprensión, Semejanzas, Cubos y Completación parece la mas adecuada. La elección de la forma corta a usar dependerá del ahorro de tiempo en la aplicación y de las funciones evaluadas en cada subprueba en función del problema en estudio.
\end{abstract}

Descriptores: Escalas de Wechsler, normas. Inteligencia, classificación. Confusión. Factore socioeconómicos.

\section{Introducción}

EIWAIS es uno de los instrumentos de evaluación intelectual del adulto mas ampliamente utilizado en la práctica clínica. El hecho que su aplicación consuma tanto tiempo ( $1 \mathrm{~h}$ aprox.) ha derivado en variados intentos para encontrar formas mas cortas. Ȧlgunos autores han optado por una selección empírica de las subpruebas mas apropiadas dependiendo de los objetivos de la evaluación ${ }^{2,10}$. Otra modalidad ha sido la búsqueda, a través de distintos procedimientos estadísticos. de la combinación de subpruebas que mejor se correlacionen con el CI total y/o CI verbal y manual ${ }^{+8,12,15}$. También se ha trabajado sobre la selección. empírica o estadística. de item al interior de cada subprueba de manera de reducir su longitud ${ }^{1+16}$. Como resultado de los distintos enfoques hoy se cuenta con varias proposiciones de formas cortas del WAIS. Mas aun. los trabajos desarrollados por Silverstein ${ }^{\text {is }} y$ Brooker y $\mathrm{Cyl}^{+}$han derivado en propuestas de tablas de equivalencia de CI total para formas cortas del WAIS-

* Estudio financiado por Nestle Foundation, Switzerland.

* Instituto de Nutrición y Tecnología de los Alimentos INTA. Universidad de Chile. Santiago

Separatas/Reprints: I. De Andraca-Casilla 138-11 Santiago 11. Chile
$\mathrm{R}$ en base a 2 y 4 subpruebas. Las distintas revisiones acerca de la utilidad de las formas cortas del WAIS concluyen, en términos generales. que estas son apropiadas cuando se requiere medir el funcionamiento intelectual global. Sin embargo, su uso no sería recomendable si la evaluación se realiza en personas en quienes se sospecha dificultades en una o mas de las funciones medidas ${ }^{13}$.

En general, las formas cortas del WAIS han sido aplicadas preferentemente en el campoclínico $0^{3,11,13}$. Sin embargo, la necesidad de encontrar formas cortas de esta prueba se extiende también al campo de la investigación, especialmente para ía medición de habilidades intelectuales ya sea como variable independiente o de confusión. Intervenciones de educación en salud, programas de estimulación precoz. programas de prevención de drogadicción son algunos ejemplos de áreas problemas donde la capacidad intelectual de los participantes es una variable de confusión que debe ser considerada tanto en el diseño de estrategias como en la evaluación de sus resultados. Sin embargo la medición de las habilidades intelectuales puede transformarse en un costo económico excesivo para la realización de um proyecto, particularmente si la escala elegida requiere para su aplicación de un tiempo largo. La elección del WAIS presenta como ventajas el sólido estudio que respalda su construcción, estandarización y elabora- 
ción de normas, la nutrida investigación posterior que avala su aplicación e interpretación y la comparabilidad con otros estudios producto de su amplia utilización a nivel internacional en la práctica clínica y estudios de investigación.

El objetivo del presente estudio es proponer una forma corta del WAIS, con bajopeso de lo cultural, para evaluar la capacidad intelectual y estimar su efecto en investigaciones referentes a poblaciones de nivel socioeconómico medio-bajo y bajo.

\section{Metodología}

\section{Antecedentes}

La muestra del presente estudio se deriva de una investigación prospectiva de terreno, realizada durante los años 1989-1992. Sus principales objetivos fueron evaluar el efecto de un cereal infantil fortificado con hierro en la prevención de la anemia durante el primer año de vida y caracterizar los efectos adversos de la anemia sobre la conducta infantil. Entre las diversas mediciones realizadas se evaluó el rendimiento intelectual de las madres, se consignaron características sociodemográficas (edad y escolaridad), y se aplicó una encuesta de nivel socioeconómico ${ }^{1}$.

Se incluyó en la investigación original lactantes de 4 meses de edad, con un peso de nacimiento mayor que 3.000 grs, sin alteraciones genéticas, neurológicas o metabólicas y sin patologías crónicas. Se excluyeron aquellos lactantes que no tuvieran cuidadoras estables $y / 0$ fueran analfabetas.

\section{Muestra}

La muestra estuvo constituida por 161 protocolos completos del WAIS, adaptación chilena realizada por Berdichewski y Herreros ${ }^{6}$. Estos protocolos fueron aplicados a madres y/o cuidadoras de lactantes de nivel socioeconómico medio bajo y bajo que reciben atención de salud en un consultorio comunitario ubicadoen el área sur oriente de Santiago de Chile. La población es homogénea en cuanto a sus características étnicas y culturales.

La edad promedio de las mujeres fue $27 \pm 6,8$ años, la escolaridad promedio $9,7 \pm 2,6$ años de estudio. En la Figura 1 se describen las distribuciones de frecuencias de estas variables.

\section{Procedimiento}

Como parte de las actividades del proyecto original, las madres fueron citadas al consultorio, donde una misma psicóloga (C.C.) administró el WAIS completo. La sesión de evaluación tuvo una duración aproximada de una hora.
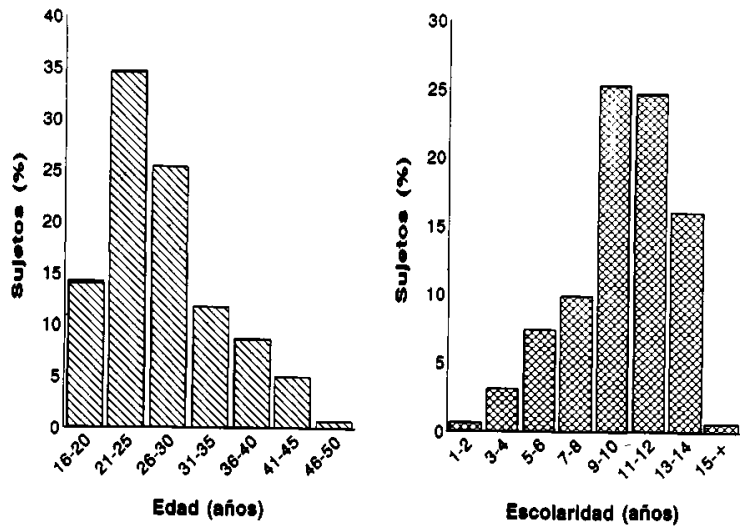

Figura 1. Distribución de frecuencia de edad y escolaridad de 161 mujeres de nivel socioeconómico medio-bajo y bajo.

\section{Análisis de los datos}

La información fue registrada en un formulario codificado e ingresada a una base de datos. Se analizaron los rendimientos en cada una de las subpruebas del WAIS y los resultados globales de las subescalas. Con el fin de seleccionar las subpruebas mas adecuadas para conformar la forma abreviada se sometió la base de datos a análisis de corrrelación de Pearson y regresión lineal. Se evaluó cinco alternativas de formas cortas del WAIS.

\section{Resultados}

El rendimiento intelectual promedio del grupo estudiado correspondio a inteligencia normal lenta y la distribución de los puntajes observados fue normal (Media:83,3; Mediana:83), observándose la moda en el rango 80-89. En la Tabla 1 se muestran los rendimientos en la Escala Total, Verbal y Manual y en la Figura 2 sus distribuciones de frecuencias.

$\mathrm{Al}$ analizar el rendimiento en las subpruebas se observó que en seis de ellas fue normal (Media \pm DS: $10 \pm 3$ ), una se encontró en el límite inferior del rango normal y cuatro se encontraron en dispersión negativa. Las subpruebas con rendimientos deficitarios fueron Comprensión (5,9 91,7$)$, Dígitos $(6,1 \pm 2,5)$, Aritmética $(6,4 \pm 1,8)$ y Símbolos $(6,6 \pm 2,0)$. El rendimiento en Información $(7,0 \pm 1,7)$ es límite, y los rendimientos

Tabla 1. Cl Total, Verbal y Manual: Promedios, desviación estándar y rangos $(n=162)$.

\begin{tabular}{cccc}
\hline Rendimientos & Cl Total & Cl Verbal & Cl Manual \\
\hline Promedio & 83,3 & 83,4 & 85,5 \\
Desviac. Estándar & 9,9 & 9,6 & 11,0 \\
Mínimo & 59 & 61 & 59 \\
Máximo & 119 & 114 & 123 \\
\hline
\end{tabular}




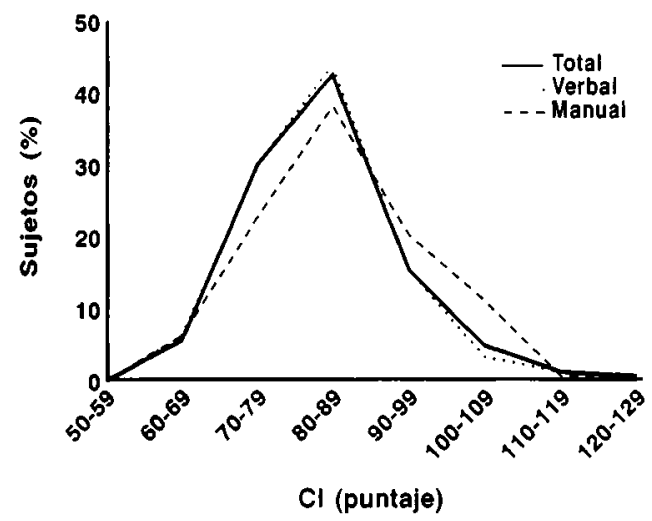

Figura 2. Distribución de frecuencia del Cl Total, Verbal y Manual de 161 mujeres de nivel socioeconómico medio-bajo y bajo.

normales corresponden a Semejanzas $(8,0 \pm 2,3)$, Vocabulario $(9,4 \pm 2,0)$, Completación $(7,9 \pm 1,9)$, Cubos $(8,7 \pm 2.7)$, Ordenación $(7,3 \pm 1,9)$ y Ensamblaje $(7,6 \pm 2,6)$.

La Tabla 2 muestra la matriz de correlaciones de las distintas subpruebas con el CI Total, Verbal y Manual. y del CI total con los CI parciales. Todas las subpruebas alcanzaron una alta relación con el CI Total, siendo las de mayor peso las de Información, Semejanzas, Vocabulario, Completación y Cubos. Del mismo modo, las subpruebas de la Escala Verbal se asociaron fuertementecon el CI Verbal y las deEscala Manual con el CI correspondiente.

Se seleccionaron tres subpruebas de cada escalaen base a un criterio conceptual (principales funciones evaluadas) y a un criterio estadístico (fuerza de la asociación con los $\mathrm{CI}$ ) y se evaluó diversas formas abreviadas del WAIS. Para la Escala Verbal se eligieron las subpruebas Semejanzas, Comprensión y Vocabulario. En la Escala Manual se eligieron Cubos, Completación y Ensamblaje. Se evaluó la relación de las tres subpruebas con el CI de la Escala respectiva, a partir de la sumatoria de los puntajes estándar obtenidos
Tabla 2. Correlaciones de las Subpruebas con $\mathrm{Cl}$ Total, Verbal y Manual $(n=162)$.

\begin{tabular}{cccc}
\hline Sub-Pruebas & Cl Total & Cl Verbal & Cl Manual \\
\hline Información & 0,76 & 0,81 & \\
Comprensión & 0,62 & 0,68 & \\
Aritmética & 0,68 & 0,71 & \\
Semejanzas & 0,76 & 0,76 & \\
Dígitos & 0,60 & 0,69 & \\
Vocabulario & 0,79 & 0,80 & \\
Símbolos & 0,60 & & 0,63 \\
Completación & 0,72 & & 0,77 \\
Cubos & 0,76 & & 0,79 \\
Ordenación & 0,60 & & 0,70 \\
Ensamblaje & 0,67 & & 0,76 \\
\hline Cl Total & & 0,92 & 0,91 \\
\hline
\end{tabular}

$g l=160 ; r=0,26, p<0,01$

en cada una de ellas. El mismo análisis se repitió utilizando combinaciones de dos subpruebas para cada Escala. La relación con el CI total se estudió considerando la sumatoria de los puntajes estándar de las 6 subpruebas, o de las respectivas combinaciones de cuatro de ellas. Los coeficientes de correlación obtenidos demonstraron una estrecha relación entre los puntajes generados en las cinco alternativas abreviadas propuestas y los coeficientes intelectuales, tantoglobales como parciales (Tabla 3).

Se estimó los puntajes de CI total a través del análisis de regresión lineal para cada una de las formas cortas. En la Tabla 4 se resumen las fórmulas, el error estándar del $\mathrm{Cl}$ estimado y el porcentaje de madres cuyos puntajes caen fuera del rango derivado del error estándar. El error estándar observado fue de $\pm 3-4$ puntos de CI, siendo el menor el observado para la altemativa con 6 subpruebas. El porcentaje de error de clasificación varía entre $3 \%$ y $7 \%$.

Tabla 3. Formas cortas del WAIS. Correlaciones con Cl Total, Verbal y Manual( $n=162)$.

\begin{tabular}{|c|c|c|c|}
\hline Alternativas & Cl Total & Cl Verbal & Cl Manual \\
\hline SEM + COM & & 0,85 & \\
\hline$S E M+V O C$ & & 0,86 & \\
\hline$S E M+C O M+V O C$ & & 0,90 & \\
\hline$C U B+C P L$ & & & 0,88 \\
\hline CUB + ENS & & & 0,88 \\
\hline$C U B+C P L+E N S$ & & & 0,92 \\
\hline$S E M+C O M+C U B+C P L$ & 0,91 & & \\
\hline$S E M+V O C+C U B+E N S$ & 0,92 & & \\
\hline$S E M+C O M+C U B+C P L$ & 0,90 & & \\
\hline$C E M+V O C+C U B+C P L$ & 0,90 & & \\
\hline$S E M+C O M+V O C+C U B+C P L+E N S$ & 0,94 & & \\
\hline
\end{tabular}

$\mathrm{SEM}=$ Semejanzas, $\mathrm{COM}=$ Comprensión, $\mathrm{CUB}=$ Cubos, $\mathrm{CPL}=$ Completación, $E N S=$ Ensamblaje, VOC = Vocabulario 
Tabla 4. Estimación del Cl total para las diferentes formas corłas del WAIS.

\begin{tabular}{lccc}
\hline Alternativas & Regresión & Error St & \% Error de clasificación \\
\hline SEM+COM+CUB+CPL & $y=44,0+1,3 x$ & \pm 4 & 3,1 \\
SEM+COM+CUB+ENS & $y=47,4+1,2 x$ & \pm 4 & 4,9 \\
SEM+VOC+CUB+CPL & $y=39,3+1,3 x$ & \pm 4 & 3,1 \\
SEM+VOC+CUB+ENS & $y=43,5+1,2 x$ & \pm 4 & 4,9 \\
SEM+COM+VOC+CUB+CPL+ENS & $y=40,6+0,9 x$ & \pm 3 & 6,8 \\
\hline
\end{tabular}

$\mathrm{SEM}=$ Semejanzas, $\mathrm{COM}=$ Comprensión, $\mathrm{CUB}=$ Cubos, $\mathrm{CPL}=$ Completación, ENS=Ensamblaje, VOC= Vocabulario.

Por último, se calculó la correlación entre CI y escolaridad, tanto para la forma completa como para las formas abreviadas, observándose en ambos casos coeficientes entre 0,60 y 0,70 .

\section{Discussión}

En primer lugar, es necesario comentar que el $78,4 \%$ de las mujeres evaluadas obtuvo rendimientos intelectuales bajo el rango normal. La mayor proporción de puntajes se ubicó en la categoría normal lento (CI:80-89). Esta observación replantea la interrogante de la adecuación de los instrumentos de evaluación. en este caso el WAIS, para la medición de grupos con características socioculturalesmas desventajadas. Aunque indudablemente es necesario desarrollar pruebas que consideren la especificidad cultural, el WAIS sigue siendo la mejor altemativa a pesar de sus reconocidas limitaciones en cuanto a sesgo cultural.

El análisis de protocolos completos del WAIS de 161 mujeres de nivel socioeconómico medio bajo y bajo permite afirmar que el uso de formas cortas es apropiado cuando el objetivo es medir la capacidad intelectual como variable de confusión. Tanto la aplicación de 4 como de 6 subpruebas arrojan conrelaciones altas, todas mayores que 0,85 entre el $\mathrm{CI}$ total y los coeficientes parciales.

De acuerdo al análisis realizado en nuestro grupo de estudio las subpruebas verbales que mostraron mayor conrelación con el CI total fueron Información. Vocabulario y Semejanzas. La decisión de no incluir Información en las alternativas de formas cortas se basó en que el rendimiento en esta subprueba presenta una fuerte influencia cultural? . Lo anterior es particularmente importante si se considera que la muestra del estudio eran mujeres de nivel socioeconómico medio bajo y bajo, con un promedio de escolaridad de 9.7 años. La subprueba Vocabulario fue seleccionada por su alta correlación con el CI total y verbal, y por su alto peso en el factor $\mathrm{G}^{7}$. La subprueba de Comprensión. en cambio, fue elegida ya que en el estudio original donde se recopilaron los protocolos del WAIS la inteligencia matema fue una variable de confusión para el desarrollo psicomotor infantil. Por ello nos pareció que la evalua- ción de las habilidades de adecuación social, medida a través de la subprueba de Comprensión, eran relevantes para la variable dependiente a pesar de tener una marcada influencia cultural y que su correlación con el CI total fuera comparativamente mas baja.

La inclusión de la subprueba de Semejanzas se basó en que esta mide razonamiento a partir de estímulos verbales, tiene un alto peso en el factor $G$ y estaríamas libre de la influencia de la culturas.

Entre las subpruebas manuales, las mas altas correlaciones con el CI total fueron las de Cubos, Completación y Ensamblaje. La elección de Cubos se basó en que también mide razonamiento, pero a partir de estímulos visuales, y estaría relativamente libre de la influencia cultural. Esta subprueba se relacionaría además con el factor organización perceptual planteado por diversos autores ${ }^{5}$. La selección de Cubos es además coincidente con otras formas cortas publicadas. En una revisión realizada por Boone ${ }^{3}$ en el año 1990, este autor señala que de seis formas cortas del WAIS-R analizadas todas incluyen la subprueba de Cubos, la que es acompañada por Vocabulario en 5 de ellas: Aritmética es incorporada en tres de las versiones, siendo las otras Información, Semejanzas y Ensamblaje. Otra razón que consideramos es la amplia difusión del CUSE (Cubos+Semejanzas) como forma corta de uso frecuente para evaluaciones clínicas de screening.

Respecto de la subprueba de Completación, esta constituiría una buena medida de recepción y memoria visual, es decir, de derivar un significado de estímulos visuales sin necesitar de la habilidad visomotora. Se ha postulado además que, al igual que Ensamblaje y $\mathrm{Cu}$ bos, sería menos influenciable por las oportunidades culturales y educativas. Se agrega a lo anterior la simpleza de su aplicación. En cuanto a la subprueba de Ensamblaje esta es una medida de expresión manual que trabaja a partir de estímulos significativos y requiere. lo mismo que Cubos, de habilidad visomanual'.

La combinación de subpruebas verbales que mejor se correlaciona con el CI verbal en nuestro grupo de estudio es Semejanzas y Vocabulario $(r: 0,86)$. Semejanzas y Comprensión alcanzan también una correlación alta $(\mathrm{r}: 0,85)$. Cuando se analiza la combinación de tres subpruebas de la escala verbal la correlación aumenta levemente $(\mathrm{r}: 0.90)$. Al examinar las subpruebas 
manuales se observa algo similar, ya que Cubos asociado con Completación o con Ensamblaje alcanzan correlaciones idénticas cuando se lo relaciona con el $\mathrm{CI}$ manual (r:0,88). Al agregar una tercera subprueba manual la relación aumenta a 0,92. Si consideramos que uno de los objetivos de buscar una forma corta es justamente el ahorrar tiempo en su aplicación, ello conduciría a la elección de la subprueba de Comprensión por sobre Vocabulario y de Completación en vez de Ensamblaje como segunda subprueba manual. La estimación del CI total a partir de estas subpruebas (Semejanzas, Comprensión, Cubos y Completación) muestra un error estándar de \pm 4 puntos de CI, lo que es razonablemente bajo. Además, según la clasificación de Kaufman las habilidades evaluadas por la subprueba de Ensamblaje son cubiertas por las subpruebas de Cubos y Completación'. Sin embargo, indudablemente el criterio de elección del número de subpruebas y de la combinación de ellas dependerá principalmente de las habilidades intelectuales mas relevantes para el objetivo de la evaluación intelectual.

Otro factor que consideramos en la selección de las subpruebas, aunque de menos importancia, es el hechoque para las mujeres de este nivel socioeconómico resulta difícil asistir solas a las sesiones de evaluación. Frecuentemente concurren acompañadas por hijos pequeños, que se resisten a permanecer alejados de sus madres durante algún tiempo, por lo que las evaluaciones muchas veces se realizan mientras ellas sostienen a un niñoen brazos. En estas situaciones la manipulación de objetos como las piezas de los puzles se ve dificultada, siendo preferible la inclusión de la subprueba de Completación. De este modo, a fin de minimizar la intervención manual de las madres sólo se seleccionó Cubos considerando su alta correlación con el CI total y el antecedente de su inclusión en la mayoría de las proposiciones de formas cortas del WAIS.

La proposición de una forma corta con 4 subpruebas formulada por Silverstein no nos parece adecuada para nuestro grupo de estudio, a pesar de contar con un sólido respaldo en cuanto a los análisis estadísticos que apoyan la selección de las subpruebas y de disponer de tablas de conversión para el CI total ${ }^{15}$. Este autor propone la inclusión de las subpruebas Vocabulario, Aritmética, Cubos y Ordenación. Por una parte, la subprueba Ordenación mostró en nuestro grupo de estudio una baja correlación con el CI total. Al mismo tiempo, es conocido que el rendimiento en Aritméticaes sensible a los niveles de ansiedad de los sujetos que se evalúan. Para las mujeres que conformaron la muestra analizada la evaluación psicológica es una situación por lo general desconocida, que genera con frecuencia elevados niveles de ansiedad. A lo anterior se agrega la inseguridad que experimentan frente a las preguntas de esta subprueba, las que son percibidas como de contenido. Este último punto es especialmente importante en las personas con baja escolaridad.
El número de subpruebas a incluir en una forma corta del WAIS ha sido discutido anteriormente en la literatura. Boone demostró en un grupo de pacientes psiquiátricos que la predicción del CI total a partir de 4 subpruebas es significativamente mejor que cuando se usan sólos dos, ya que el error estándar del CI estimado disminuye ${ }^{3}$. De acuerdo a los resultados que hemos presentado esclaro que la aplicación de 6 subpruebas no es significativamente mejor que la aplicación de 4, por lo que no existirian fundamentos para hacerlo.

Analizamos una altenativa de forma corta del WAIS con 6 subpruebas y cuatro alternativas con 4 subpruebas. Todas ellas resultan adecuadas para reemplazar la escala completa, mostrando una alta correlación tanto con el CI total como con los CI verbal y manual y un error estándar de estimación del CI total no mayor de 4 puntos. Consideramos que un criterio importante en la elección de una de las alternativas abreviadas para la evaluación de rendimiento intelectual es la economía de tiempo en la aplicación. Sin embargo es de similar importancia que la forma elegida incluya subpruebas que midan habilidades relevantes para el problema en estudio, siendo algo a definir de acuerdo al criterio de los investigadores. En el caso de estudios donde el objetivo es evaluar el efecto de la inteligencia materna en el desarrollo cognitivo del niño, nosotros hemos optado por la forma corta que incluye Comprensión, Semejanzas, Cubos y Completación, ya que estas pruebas evaluarían las habilidades que están más involucradas en el desarrollo infantil.

En nuestra experiencia la inclusión de la variable inteligencia materna ha resultado altamente relevante para la interpretación de observaciones relativas al desarrollo infantil. El contar con formas cortas del WAIS, que surgen del análisis de protocolos aplicados a mujeres latinoamericanas puede ser una ayuda al decidir en futuras investigaciones la evaluación de habilidades intelectuales en estudios donde el gran tamaño muestral requerido puede llevar a los investigadores a desecharla no por asignarle poca importancia sino por la elección obligada que deben hacer en términos de los límites de los diseños y la adecuación a la disponibilidad de recursos económicos para su ejecución. Las ventajas de utilizar formas cortas del WAIS en vez de otras escalas mas breves son su reconocida validez y confiabilidad, y la comparabilidad con otros estudios por ser un instrumento ampliamente difundido a nivel internacional.

ANDRACA, I. de et al. [The use of short forms of WAIS for the assessment of the intelligence of persons of low socioeconomic status]. Rev. Saúde Pública, 27:334-9, 1993. A WAIS-S short form for the assessment of intelligence as a confounding variable is proposed. A number of 161 complete WAIS-S protocols, relating to women of lower-middle and low socioeconomic status were analysed. Six subtests (Social Comprehension, Similarities, Vocabulary, Block Design, Picture Completion 
and Object Assembly) were chosen using two criteria: specific functions evaluated by each subtest and correlation coefficients relating IQ to the subtests. Five WAIS-S short forms were evaluated: one consisting of six subtests and the others with different combinations of four of them. Data showed that they were all appropriate for the measurement of intelligence as a confounding variable; the correlation coefficients between short forms and total IQ ranged from 0.90 to 0.94 . The standard errors of the short forms for the estimation of total IQ were of 3 or 4 points, and 3 to $7 \%$ of the subjects were wrongly classified. When maternal intelligence had to be measured in relation to infant development the short form that included Social Comprehension, Similarities, Block Design and Picture Completion seemed the most appropriate. Nevertheless when choosing a WAIS-S short form not only economy of time but also the specific relevant functions assessed in each subtest must be considered.

Keywords: Wechsler scales, standards. Intelligence, classification. Confusion. Socioeconomic factors.

\begin{abstract}
ANDRACA, I. de et al. Avaliação de inteligência por meio de versão abreviada de WAIS para população de baixo nível socioeconômico. Rev. Saúde Pública, 27: 334-9, 1993. Propõe-se uma versão abreviada da forma WAIS para a avaliação da inteligência em estudos da população. Analisaram-se 161 protocolos completos do WAIS, aplicados a mulheres de nível socioeconômico baixo, e estudaram-se as correlações entre as diferentes subprovas e o coeficiente de inteligência (QI) total, o QI verbal e o QI manual. Selecionaram-se seis subprovas (compreensão, semelhanças, vocabulário, cubos, complemento e montagem) conforme os critérios: correlaçôes observadas e funções avaliadas em cada subprova. A análise de uma versão com 6 subprovas e 4 versões com 4 subprovas demonstram que todas elas são adequadas para a avaliação da inteligência como variável de confusão. $O$ erro padrão para a estimação do QI varia entre 3 e 4 pontos e o erro de classificação é de 3 a $7 \%$. Quando se avalia a inteligência materna como variável de confusão do desenvolvimento infantil, a versãoque combina compreensão, semelhanças, cubo e complemento pareceu a mais adequada. A eleição da versão a ser utilizada deverá basear-se na maior eficiência do tempo de aplicação e das funções avaliadas pelas subprovas, tendo em vista o problema em estudo.
\end{abstract}

Descritores: Escalas de Wechsler, normas. Inteligência, classificação. Confusão. Fatores socioeconômicos.

\section{Referências Bibliográficas}

1. ALVAREZ, M.L.; WURGaFF, F.; SALAZAR, M.E. Mediciones del nivel socioeconómico bajo urbano en familias con lactante desnutrido. Arch. Latinoam. Nutr. 32: 650-62, 1982.

2. BRITTON,P.G.\& SAVAGE, R.D. A short form of the WAIS for the use with the aged. Brit.J.Psychiatry, 112: 417-8, 1966

3. BOONE, D.E. Short forms of the WAIS-R with psychiatric inpatients: a comparison of techniques. J.Clin.Psychol., 46: $197.200,1990$

4. BROOKER, B.H. \& CYR, J.J. Tables for clinician to use to convert WAIS-R short forms. J.Clin.Psychol., 42: 982-5, 1986.

5. COHEN, J. The factorial structure of the WAIS between early adulthood and old age. J.Consult.Clin.Psychol., 21: 283$90,1957$.

6. HERMOSILLA, M. Escala de Inteligencia de Wechsler para adultos (WAIS): mamual. Santiago, Pontificia Universidad Católica de Chile, Escuela de Psicología, Chile, 1982.

7. HERMOSILLA, M. Un modelo de análisis del WAIS. Santiago, Escuela de Psicología, Pontificia Universidad Católica de Chile, 1988.

8. JONES, R.L. Validities of short WAIS batteries. J.Consult.Clin.Psychol., 31: 103, 1967.

9. KAUFMAN, A.S. Psicometría razonada con el WISC.R. México. Editorial El Manual Modemo, 1979.

10. LEVY,P. Short form tests: a methological review. Psychol.Bull., 69: 410-16, 1968.

11. PIETERS, H.C. \& SIEBERHAGEN, J.J. Evaluation of two shortened forms of SAWAIAS with three diagnostic groups. J.Clin.Psychol., 5: 809-15, 1986.

12. REYNOLDS, C.; WILSON, V.; CLARK, P. A four test short form of the WAIS-R for clinical screening. Clin Neuropsychol., 42: 982-6, 1986.

13. RYAN, J.J.; LARSEN, J.;PRIFITERA, A. Validity of two-and four-subtest short forms of the WAIS-R in a psychiatric sample. J. Consult.Clin.Psychol., 51: 460. 1983.

14. SATZ, P. \& MOGEL. S. An abbreviation of the WAIS for clinical use. J.Clin.Psychol., 18: 77-9, 1962.

15. SILVERSTEIN, A.J. Two and four subtest short forms of the Wechsler Adult Intelligence Scale-Revised. J.Consult.Clin.Psychol., 50: 415-8, 1982.

16. VINCENT, K.R. The modified WAIS: and altemative to short forms. J.Clin.Psychol., 35: 624-5, 1979.

Recebido para publicação em 2.12.1992

Reapresentado em 14.6.1993

Aprovado para publicação em 13.7.1993 